Isotani, S., McLaren, B.M.,\& Altman, M. (2010). Towards intelligent tutoring with erroneous examples: A taxonomy of decimal misconceptions. In V. Aleven, J. Kay, J. Mostow (Eds.), Proceedings of the 10th International Conference on Intelligent Tutoring Systems (ITS-10), Lecture Notes in Computer Science, 6094 (pp. 346-348). Berlin: Springer.

\title{
Towards Intelligent Tutoring with Erroneous Examples: A Taxonomy of Decimal Misconceptions
}

\author{
Seiji Isotani ${ }^{1}$, Bruce M. McLaren ${ }^{1}$, Max Altman ${ }^{2}$ \\ ${ }^{1}$ Human-Computer Interaction Institute, Carnegie Mellon University, PA, USA \\ ${ }^{2}$ Gila Ridge High School, AZ, USA \\ sisotani@cs.cmu.edu,bmclaren@cs.cmu.edu,max.altman@vanderbilt.edu
}

\begin{abstract}
In the mathematics domain of decimals, students have common and persistent misconceptions. These misconceptions have been identified, studied, and published by many researchers, spanning over 80 years of time. However, no paper discusses and brings together all of the identified misconceptions. This paper presents an initial taxonomy of decimal misconceptions, summarizing the results of past work. We also discuss the potential use and benefits of such a taxonomy in supporting the development of intelligent tutors that use erroneous examples as a learning tool for middle-school math students.
\end{abstract}

Keywords: Decimals, misconceptions, intelligent tutors, erroneous examples

\section{Introduction}

The understanding and correct use of decimals is a foundational topic necessary for understanding more advanced mathematical topics. However, past research has indicated that learning decimals is very difficult, leaving students with a variety of misconceptions that often persist into adulthood. Through an extensive math education literature review, covering over 40 published papers and extending as far back as 1928 [e.g., 1,2,4,5,7,8], we found that most past work addresses either a single misconception or a small set of related misconceptions. In other words, the knowledge about the problems students, and even adults, have with decimals, and the means by which these misconceptions can be addressed, is spread over a variety of published papers. There is no single blueprint or guide to how students struggle with decimals and how educational technology can be used to overcome these struggles.

As a step toward addressing this issue, this paper presents a preliminary and partial taxonomy of decimal misconceptions derived from our literature review. In addition, we discuss our intended use of the taxonomy to support the development of an intelligent tutor and erroneous examples to address students' decimal misconceptions.

\section{Taxonomy of Decimal misconceptions}

The benefits of having a taxonomy of decimal misconceptions are, on one hand, to provide an overview of the common problems that students have while working with decimals and, on the other hand, to gain insight into selecting appropriate and effective instructional strategies to help ameliorate misconceptions. Decimals treated as whole numbers or fractions [8] and incorrect beliefs such as "multiplication makes bigger" and "division makes smaller" $[2,4]$ are examples of common misconceptions. 
Our literature survey shows that there is no single paper that presents decimal misconceptions in a comprehensive manner. The closest to comprehensive coverage is a paper by Stacey et al [7]. Our work extends the efforts of Stacey et al. by including other research used to construct a taxonomy that covers the most common misconceptions found in previous research.

Much of the past work focuses on how prior knowledge of other areas of math, such as fractions, whole numbers and negative numbers, can interfere with understanding decimals. Figure 1 shows a section of the decimal misconception taxonomy related to prior knowledge. Individual misconceptions have been given short, mnemonic names for easy remembrance (e.g., Decimals misconstrued as NEGative numbers is "Negz"). The complete taxonomy also includes misconceptions related to operations (e.g., multiplication, as in "multiplication makes bigger"). The taxonomy illustrates that students often have misconceptions based on pre-existing knowledge and prior learning. For example, researchers have empirically shown that some students believe that shorter decimals are larger because of a confusion with prior learning of fractions (e.g. $0.2>0.25$ because $1 / 2>1 / 25$ ). Other students believe that longer decimals are larger $(0.25>0.7)$ because they confuse decimals with prior learning of whole numbers (e.g., $0.25>0.7$ because $25>7$ ).

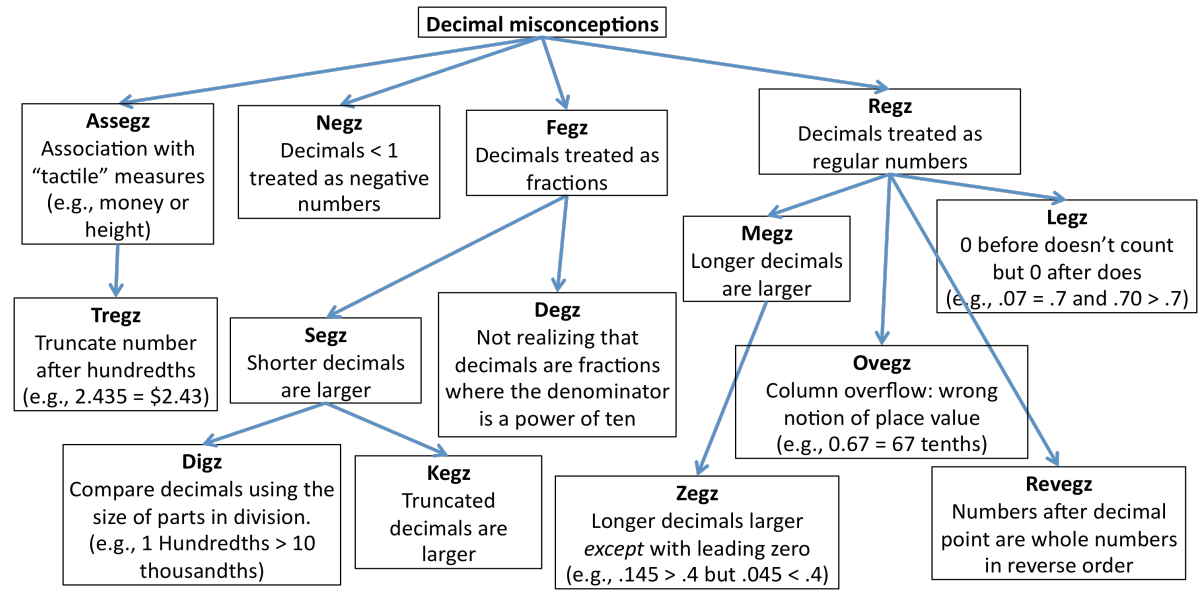

Figure 1. Part of the misconception taxonomy related to students' previous knowledge

By organizing and relating misconceptions we provide a unique resource that can be useful both for teachers and fro ITS developers to prepare appropriate educational resources to support learning.

Currently we are working on the development of an intelligent tutor that will use this taxonomy as part of its user model, one that associates students' mistakes with the corresponding misconception(s). Accordingly, we can then adapt the feedback (hints, next problems, concrete examples, etc.) to help students better understand the concept of decimals and address the particular misconceptions that led them to internalize the incorrect behavior/thinking.

Another key use of our taxonomy is the creation of an erroneous example-based tutor. An erroneous example (ErrEx) is a step-by-step problem solution in which one or more of the steps are incorrect. Past work has shown that presenting students with 
errors is valuable, as long as students are not pure novices in the problem domain $[3,9]$. Through the use of ErrEx within an intelligent tutor we will give students an opportunity to (a) compare correct solutions with incorrect ones; (b) help them understand why a given solution is wrong; and finally, (c) guide them to self explain incorrect solutions. Such an approach we believe will encourage critical thinking and motivate reflection and inquiry. This three-step process with erroneous examples is also consistent with Stellan Ohlsson's theory on learning from performance errors [6].

The erroneous examples-based tutor briefly discussed in this paper will eventually be freely available through MathTutor (webmathtutor.org), a website to help middle school children learn math with intelligent tutors. A description of our project (AdaptErrEx) is found at www.cs.cmu.edu/ bmclaren/projects/AdaptErrEx/.

\section{Conclusions}

We have discussed an initiative to create a taxonomy of decimal misconceptions. The effort has focused on gathering the findings from math education literature, spanning over 40 published papers, into a single representation. Currently, we are using this taxonomy to drive the creation of an intelligent tutor that will identify student misconceptions and support learning. We also emphasize the use of ErrEx, rarely used by math teachers but potentially quite helpful to student learning. We believe that ErrEx presented to students in an intelligent and adaptive fashion can provide the opportunity to find and reflect upon errors in a way that will lead to deeper and more robust learning. Our future work will investigate the benefits of an ErrEx-based tutor in supporting the learning of decimals.

\section{References}

1. Brueckner, L.J.: Analysis of Difficulties in Decimals. Elementary School Journal, 29, pp. 32--41 (1928)

2. Graeber, A., Tirosh, D.: Multiplication and division involving decimals: Preservice elementary teachers' performance and beliefs. Journal of Mathematics Behavior, 7, pp. 263--280 (1988)

3. Grosse, C.S., Renkl, A.: Finding and fixing errors in worked examples: Can this foster learning outcomes? Learning and Instruction, 17(6), pp. 612--634 (2007)

4. Hiebert, J.: Mathematical, Cognitive, and Instructional Analyses of Decimal Fractions. Chapter 5, pp. 283--322, Lawrence Erlbaum, New Jersey (1992)

5. Irwin, K.C.: Using everyday knowledge of decimals to enhance understanding. Journal for Research in Mathematics Education, 32(4), pp. 399--420 (2001)

6. Ohlsson, S. Learning from performance errors. Psychological Review, 103(2), pp. 241-$262(1996)$

7. Stacey, K., Helme, S., Steinle, V.: Confusions between decimals, fractions and negative numbers. 25th Conference of the International Group for the Psychology of Mathematics Education, Vol. 4, pp. 217--224 (2001)

8. Resnick, L. B., Nesher, P., Leonard, F., Magone, M., Omanson, S., Peled, I.: Conceptual bases of arithmetic errors: The case of decimal fractions. Journal for Research in Mathematics Education, 20(1), pp. 8--27 (1989)

9. Tsovaltzi, D., Melis, E., McLaren, B.M., Dietrich, M., Goguadze, G., Meyer, A-K.: Erroneous examples: A preliminary investigation into learning benefits. In Cress, U., Dimitrova, V., Specht, M. (eds.) EC-TEL 2009. LNCS, vol. 5794, pp. 688--693. Springer, Heidelberg (2009) 